\title{
Las políticas públicas de igualdad de género en México
}

\section{Public Policies for Gender Equality in Mexico}

\author{
María FeRnANDa SánChez DÍAZ ${ }^{1}$
}

Resumen: El presente artículo pretende visibilizar la deficiencia de las políticas públicas instrumentadas para alcanzar la igualdad sustantiva que continúa siendo un anhelo de las mujeres en la gran mayoría de los países, como sucede en el caso de México, país que cuenta con un marco jurídico importante en materia de igualdad, así como con instituciones enfocadas a trabajar en la igualdad entre mujeres y hombres.

Palabras clave: Políticas públicas, igualdad, equidad y género.

Abstract: This article aims to highlight the deficiency of public policies implemented to achieve substantive equality that continues to be a desire of women in the vast majority of countries, as in the case of Mexico, a country that has an important legal framework in terms of equality, as well as with institutions focused on working on equality between women and men.

\footnotetext{
${ }^{1}$ Doctora en Derecho por la Universidad Nacional Autónoma de México, Máster en Protección Internacional de los Derechos Humanos por la Universidad de Alcalá, España, Maestra en Derecho por la Universidad Nacional Autónoma de México, Licenciada en Derecho por la Universidad Nacional Autónoma de México, Profesora de Carrera de Tiempo Completo de la Universidad Nacional Autónoma de México.
} 
Keywords: Public policy; Equality; Gender.

Recibido: 16.6.2019 Aceptado: 14.1.2020

\section{Sumario}

\section{Las políticas públicas}

2. Conceptos en materia de igualdad de género

\section{La igualdad de género en el mundo}

\section{La igualdad de género en México}

5. Las políticas públicas de igualdad de género en México

6. Conclusiones

\section{Las políticas públicas}

Las políticas públicas han sido el instrumento utilizado por los Estados para buscar el desarrollo socioeconómico que contribuya al bien común. En este sentido, nos podríamos remontar al Welfare State que surgió en Reino Unido durante la Segunda Guerra Mundial, de manera contraria al sistema que imperaba en el imperio alemán.

Con el Welfare State, "a largo plazo, el crecimiento económico está en el origen del Estado protector; sin embargo, su principal efecto ha consistido en modificar las condiciones demográficas durante el último siglo y cambiar asimismo el ritmo de los programas sociales en marcha" (Meny, 1992, 22).

En este mismo sentido, Wilensky asentó que "el Estado debe asegurar la 'reproducción social' que requieren las necesidades del capitalismo: es decir, asegurar la formación, la vivienda y la 
protección social de los trabajadores y de sus familias (al mismo tiempo que garantiza la ley y el orden)" (Meny, 1992, 22).

Ahora bien, para la instrumentación de las políticas públicas se requiere de recursos económicos que sean administrados a través del gasto público, el cual, a diferencia del gasto privado, es ejercido estrictamente por las autoridades y "tiene un fin único y propio que, como ya se dijo, en esencia es respetar y hacer respetar los derechos individuales y satisfacer los derechos sociales" (Burgoa, 2014, 203).

A través de las políticas públicas se debe buscar un estado de bienestar para la sociedad. Anteriormente, a los administradores del Estado solamente les interesaban los ciudadanos que representaban un beneficio para ellos; es decir, quienes se encontraban en la posibilidad de votar por los políticos. El resto de la sociedad era simplemente ignorada. Actualmente, los cambios de régimen, el reconocimiento de mayores derechos humanos y las demandas de una sociedad cada vez más informada, organizada y demandante han obligado al Estado a instrumentar políticas públicas con distintos enfoques, como sucede con la igualdad de género.

La cuestión principal con las políticas públicas radica en su eficacia, que depende de aspectos como su discusión respecto del contexto en el que se desarrollen. Ello será un reflejo del interés verdadero por generar cambios, o revelará si se trata solamente de una cuestión de reconocimiento e interés político-personal o partidista. De igual forma impactará la manera en que se apliquen estas políticas, y ese es uno de los grandes problemas ante los que se enfrentan, pues los partidos políticos, que a través de sus representantes pueden tener en consideración diversas ideas para combatir alguna situación problemática, pueden no contar con una visión real y tangible para la instrumentación de esas políticas, las cuales desde ese momento podría decirse que fracasaron antes de nacer.

Es en este terreno que nos parece indispensable que se abran fronteras mediante el estudio de políticas que ofrezcan una 
probabiilidad razonable de iluminar, con el grado de especificidad y de atención necesarios para detectar interacciones a lo largo del tiempo, los muchos aspectos de aquellos grandes temas que quedan ocultos para las lentes inevitablemente más estáticas y agregadas de otros enfoques, también necesarios pero más "estructurales" (Oszlack, 1995, 103).

En este sentido, si nos enfocamos en la cuestión de la igualdad de género y tomamos como referencia la importancia que solo se le daba a las personas que tenían cierto poder en las decisiones del Estado, notamos que las mujeres no se encontraban en tal situación; todo lo contrario, eran prácticamente inexistentes en lo referente a las decisiones de Estado y las políticas públicas. Las mujeres estaban limitadas en el ejercicio de sus derechos; solamente se les concedía la capacidad de goce mas no de ejercicio, y este último debía ser a instancia de su marido, como sucedía en el caso de las sucesiones.

La actuación de las mujeres se restringía a realizar actividades en "el servicio doméstico y el cuidado de la prole, mientras que el varón puede ver realizados sus intereses y su ambición en todos los demás campos de la productividad humana" (Millet, 1970, 72). En un sentido similar, Carole Pateman señala que "los hechos fisiológicos de la maternidad y la paternidad nunca fueron socialmente vistos de la misma manera, la paternidad ha significado engendrar. La paternidad ha significado el rol primario, esencial y creativo" (Pateman, 1995, 53).

Este panorama patriarcal, donde la mujer se ha visto disminuida en cuanto a las actuaciones que puede desarrollar en la sociedad, ha sido propiciado, en gran parte, por la estructura familiar que prolonga la figura patriarcal. De acuerdo con Kate Millet, "el patriarcado gravita sobre la institución de la familia. Ésta es, a la vez, un espejo de la sociedad y un lazo de unión con ella; en otras palabras, constituye una unidad patriarcal dentro del conjunto del patriarcado" (Millet, 1970, 83). 
Las mujeres, en muchas regiones y por siglos, han sido cosificadas; deshumanizadas e ignoradas jurídicamente como personas. Sin embargo, se ha ido avanzando en un empoderamiento jurídico de las mujeres, mas no social, porque ello implicaría modificar los patrones culturales y económicos de la sociedad. En este sentido, el derecho ha servido solamente como un discurso normativo para dar la apariencia de que las mujeres ya son reconocidas realmente por el Estado, aunque esta normatividad sea letra muerta.

En los patriarcados contemporáneos, la prioridad de derecho del varón se ha visto recientemente menoscabada por la concesión del divorcio, la ciudadanía y la propidad a la mujer. No obstante su equiparación con un mero objeto poseído sigue manifestándose en la pérdida del apellido, la obligación de residir en el domicilio del marido y la presunción legal de que el matrimonio supone, por parte de la esposa, el cuidado del hogar y el consorcio (sexual) a cambio de protección económica (Millet, 1970, 86).

De esta forma se observa que mientras en el pasado contar con políticas públicas de género no era una prioridad para las autoridades, actualmente es una exigencia mundial. Sin distinguir entre fronteras, razas, edad, riqueza o pobreza, la gran mayoría de las mujeres en algún momento de su vida han sido víctimas de discriminación por el simple hecho de ser mujeres, ya sea en el ámbito familiar, social o laboral, entre otros, o han experimentado los conocidos micromachismos, que son difíciles de detectar y que incluso cometen algunas mujeres en contra de otras mujeres, al ser una costumbre social normalizada y tolerada desde el núcleo familiar, lo que ha permitido que se exteriorice a los diversos ámbitos de la vida pública, incluso por el propio Estado en el actuar de sus instituciones.

Otro de los problemas que enfrenta el éxito de las políticas públicas es la falta de continuidad ante los cambios de gobierno. Ernesto Stein y Mariano Tommasi explican por qué las experiencias con las políticas varían en las diversas jurisdicciones: 
- Mientras algunos países mantienen políticas encaminadas en la misma dirección básica durante periodos prolongados, creando así un entorno estable y predecible, otros las modifican frecuentemente, a menudo con cada cambio de gobierno.

- Mientras algunos países adaptan ágilmente sus políticas a los cambios de las circunstancias externas o innovan cuando las políticas pierden eficacia, otros reaccionan con lentitud $o$ gran dificultad, aferrándose a políticas inapropiadas por demasiado tiempo.

- Mientras algunos países aplican y hacen cumplir eficazmente las políticas promulgadas por el parlamento o el ejecutivo, otros se toman un tiempo considerable para ponerlas en marcha o nunca lo hacen de manera eficaz.

- Mientras algunos países adoptan políticas concentradas en el interés público, otros promulgan políticas abundantes en tratamientos especiales, lagunas legales y excepciones (Stein, 2006, 395).

En México, esta falta de continuidad en las políticas es una constante, lo que no brinda una atmósfera de estabilidad y permite que los problemas se sigan agravando, pero ahora con distintas vertientes. Por ello, el tema de la igualdad entre mujeres y hombres en México continúa siendo un anhelo difícil de consolidar, y la igualdad sustantiva sigue siendo una obligación lejos de cumplirse.

De acuerdo con Ernesto Stein y Mariano Tommasi, las políticas públicas deben considerar los siguientes aspectos:

- Estabilidad. La medida en la que las políticas son estables en el tiempo. Sin embargo, no se debe confundir estabilidad con rigidez. Contar con políticas estables no significa que las políticas no puedan cambiar en lo absoluto, sino más bien que los ajustes respondan a cambios de las condiciones económicas o a defectos de las políticas, más que a caprichos políticos. 
- Adaptabilidad. La medida en la que es posible ajustar las políticas cuando estas fallan o cuando las circunstancias cambian. Esta es la otra cara de la moneda de la estabilidad. Las políticas deben ser lo suficientemente flexibles como para responder a las sacudidas o cambiar con los tiempos, pero no tanto como para que sean susceptibles de manipulaciones políticas.

- Coherencia y coordinación. La medida en la que las políticas se corresponden con politicas afines y son producto de medidas bien coordinadas entre las personas que participan en su diseño y aplicación. En vista de la cantidad de piezas dinámicas que integran el proceso de diseño de políticas y la diversidad de intereses e incentivos presentes, la falta de coordinación puede ser intencional o involuntaria.

- La calidad de implementación y ejecución. Más de una política bien concebida ha quedado abandonada en el camino a causa de una aplicación precaria. Cuán bien se aplique y se haga cumplir una política depende mucho de la existencia de una burocracia capaz e independiente y de un poder judicial poderoso.

- Orientación hacia el interés colectivo. La medida en la que las políticas procuran el interés público. ¿Promueven las políticas el bienestar general o canalizan beneficios privados hacia individuos, facciones o regiones específicos? Esta dimensión guarda una estrecha relación con la desigualdad, ya que los miembros de la élite tienen la influencia económica y política para modificar decisiones políticas y hacer que los favorezcan, apartándolas del interés general.

- Eficiencia. La medida en que las políticas responden a la asignación más productiva de recursos escasos. Esta característica va de la mano con el grado en el que se tiene en cuenta al público, ya que favorecer a sectores específicos en detrimento del interés general constituye por lo general una asignación ineficiente de recursos (Stein, 2006, 397). 
En materia de políticas de igualdad de género, el logro que se ha alcanzado ha sido el de contar con un presupuesto etiquetado para su instrumentación. Sin embargo, el problema aquí es su correcta planeación y aplicación, así como un adecuado seguimiento, evaluación y exigencia hacia las autoridades. La Comisión de Igualdad de Género de la Cámara de Diputados, el Instituto Nacional de las Mujeres y la Secretaría de Hacienda y Crédito Público dan seguimiento trimestral al cumplimiento de indicadores y ejercicio del presupuesto, a lo que se agrega la facultad de la Comisión Nacional de los Derechos Humanos para observar la política nacional en materia de igualdad entre mujeres y hombres. No obstante la existencia de todos estos mecanismos de control y vigilancia, aún no se cuentan con sistemas que permitan evaluar cualitativamente el impacto de las políticas públicas.

\section{Conceptos en materia de igualdad de género}

En primera instancia, es importante entender qué es la igualdad y qué es la equidad. Estos son dos conceptos distintos que se interrelacionan, pero que son en gran medida confundidos por la sociedad y también por las autoridades, lo que genera que ninguno de los dos se lleve a cabo.

La discusión conceptual data de décadas, partiendo desde el aspecto multuculturalista, donde se ha llegado a preguntar “iqué se necesitaría para que todos pudiesen participar como iguales? $i \mathrm{La}$ democracia multicultural exige igualdad social? ¿Equidad entre género? ¿Equidad entre regiones? ¿Equidad entre grupos étnicos? ¿Ausencia de dominación y subordinación en el sistema?" (Fraser, 1995, 35-36).

Este desconocimiento de lo que significa, o los alcances que tienen uno y otro concepto necesariamente impacta en el diseño de las políticas públicas y en los resultados que se requieren, toda vez que no existe una orientación ad hoc para alcanzar la igualdad sustantiva 
y la equidad entre los géneros, atendiendo a la particularidad de cada uno; es decir, a los distintos factores que pueden generar una vulnerabilidad que implicaría reorientar una política pública.

La Ley General para la Igualdad entre Mujeres y Hombres afirma que por "igualdad de género" debe entenderse la "situación en la cual mujeres y hombres acceden con las mismas posibilidades $\mathrm{y}$ oportunidades al uso, control y beneficio de bienes, servicios y recursos de la sociedad, así como a la toma de decisiones en todos los ámbitos de la vida social, económica, política, cultural y familiar” (Ley General para la Igualdad entre Mujeres y Hombres, 2016, Art. 5, fracción, IV).

En cambio, el término "equidad de género" no está contenido en la Ley General para la Igualdad entre Mujeres y Hombres, y se ignora la razón por la cual fue omitido. Sin embargo, sí encontramos una definición en la Ley del Instituto Nacional de las Mujeres, que la entiende como el

principio conforme al cual hombres y mujeres acceden con justicia e igualdad al uso, control y beneficios de los bienes y servicios de la sociedad, incluyendo aquéllos socialmente valorados, oportunidades y recompensas, con la finalidad de lograr la participación equitativa de las mujeres en la toma de decisiones en todos los ámbitos de la vida social, económica, política, cultural y familiar (Ley del Instituto Nacional de las Mujeres, 2015, Art. 5).

De estos dos conceptos, es preciso entender que la igualdad no implica que mujeres y hombres deban ser tratados de manera idéntica, sino que tanto unas como otros puedan tener el mismo acceso a las oportunidades en la vida pública y privada. Actualmente, se está trabajando en incorporar la perspectiva de género, lo que obliga a actuar atendiendo a las diferencias de hombres y mujeres, siendo estas últimas quienes a lo largo de la historia han tenido una participación más limitada en la vida pública principalmente. 
Ahora bien, el considerar la perspectiva de género y la necesidad de empoderar a las mujeres en los distintos ámbitos ha obligado al Estado, en todos los rincones del mundo, a instrumentar lo que se conocen como "acciones afirmativas", que de acuerdo con la Ley General para la Igualdad entre Mujeres y Hombres son el "conjunto de medidas de carácter temporal correctivo, compensatorio y/o de promoción, encaminadas a acelerar la igualdad sustantiva entre mujeres y hombres" (Ley General para la Igualdad entre Mujeres y Hombres, 2016, Art. 5, I).

A las acciones afirmativas también se les conoce como "discriminación positiva", y van encaminadas a revertir la discriminación que sufrió la mujer durante muchos años, particularmente para acceder a puestos de trabajo, o también en la vida política, al establecer una cuota de género en los cargos de elección popular.

En cuanto al término "perspectiva de género", la Ley General para la Igualdad entre Mujeres y Hombres nos dice que es

la metodología y los mecanismos que permiten identificar, cuestionar y valorar la discriminación, desigualdad y exclusión de las mujeres, que se pretende justificar con base en las diferencias biológicas entre mujeres y hombres, así como las acciones que deben emprenderse para actuar sobre los factores de género y crear las condiciones de cambio que permitan avanzar en la construcción de la igualdad de género (Ley General para la Igualdad entre Mujeres y Hombres, 2016, Art. 5 VI).

Respecto a la igualdad sustantiva, mucho se ha hablado sobre que debe existir la igualdad entre mujeres y hombres, pero esto quedaba en el plano del mero discurso al no existir normativamente, de modo que no se obligaba a nadie a garantizar esta igualdad. Con la promulgación de la Ley General para la Igualdad entre Mujeres y Hombres a la que hemos venido haciendo referencia, se normativiza ese discurso, es decir se formaliza, pero el problema ahora es que esta 
igualdad se lleve a la práctica diaria, es decir, que sea una realidad tangible para todas las mujeres. En este sentido nos estamos refiriendo a la igualdad sustantiva, que es en lo que debe trabajar el Estado a través de las políticas públicas de género que contruyan las condiciones de igualdad necesarias.

En este sentido, la ley antes referida nos proporciona lo que debe considerarse como igualdad sustantiva, entendiéndola como "el acceso al mismo trato y oportunidades para el reconocimiento, goce o ejercicio de los derechos humanos y las libertades fundamentales" (Ley General para la Igualdad entre Mujeres y Hobres, 2016, Art. 5, V). Al respecto, Ana Laura Rodríguez Gustá, en su obra “Las políticas sensibles al género: variedades conceptuales y desafíos de intervención" advierte que las acciones afirmativas que llevan a cabo los Estados "promueven la presencia femenina en los lugares decisorios del Estado” (Rodríguez, 2008, 2).

De igual forma, es importante conocer lo que es la "transversalidad", ya que en México es un mandato presidencial la transversalización de la perspectiva de género en toda la actuación de la Administración Pública Federal. La Ley General para la Igualdad entre Mujeres y Hombres nos dice que la transversalidad "es el proceso que permite garantizar la incorporación de la perspectiva de género con el objetivo de valorar las implicaciones que tiene para las mujeres y los hombres cualquier acción que se programe, tratándose de legislación, políticas públicas, actividades administrativas, económicas y culturales en las instituciones públicas y privadas" (Ley General para la Igualdad entre Mujeres y Hombres, 2016, Art. 5, VII).

Otro concepto relevante en materia de políticas públicas de igualdad de género es el de "acciones afirmativas", entendiéndolas según la Ley General para la Igualdad entre Mujeres y Hombres como "el conjunto de medidas de carácter temporal correctivo, compensatorio y/o de promoción, encaminadas a acelerar la igualdad sustantiva entre mujeres y hombres" (Ley General para la Igualdad entre Mujeres y Hombres, 2016, Art. 5, I). Estas acciones son el 
mecanismo más importante para consolidar la igualdad sustantiva, así como para remediar las injusticias generadas por la discriminación de la cual han sido objeto las mujeres por el hecho de ser mujeres.

Como puede apreciarse, en la actualidad ya se cuenta con las definiciones necesarias para poder llevar a cabo acciones en pro de la igualdad de género en México y que ésta sea una realidad y no solamente un juego político que no tendrá resultados favorables y sí un incremento en los indicadores de violencia de género. Sin embargo, estas acciones requieren de voluntad política para su eficacia, y de la participación ciudadana en su totalidad.

\section{La igualdad de género en el mundo}

El movimiento dirigido a consolidar la igualdad sustantiva entre mujeres y hombres es internacional. Desde las organizaciones de la sociedad civil hasta la Organización de las Naciones Unidas han hablado a favor de combatir la discriminación de género.

La desigualdad de género afecta a más del cincuenta por ciento de la población mundial. Esta desigualdad se agrava aún más cuando la mujer es parte de uno de los considerados grupos vulnerables, como el tener alguna discapacidad física, pertenecer a un determinado grupo racial, no contar con recursos económicos o con estudios, entre otros factores que convierten la situación de esas mujeres en un panorama aún más desalentador.

En el año 1995 se firmó la Declaración y Plataforma de Acción de Beijing en la Cuarta Conferencia Mundial sobre la Mujer. Por las declaraciones que allí se realizaron, se considera importante hacer una breve transcripción de algunas de ellas:

3. Decididos a promover los objetivos de igualdad, desarrollo y paz para todas las mujeres del mundo, en interés de toda la humanidad, 
4. Reconociendo las aspiraciones de las mujeres del mundo entero y tomando nota de la diversidad de las mujeres y de sus funciones y circunstancias, haciendo honor a las mujeres que han allanado el camino, e inspirados por la esperanza que reside en la juventud del mundo,

5. Reconocemos que la situación de la mujer ha avanzado en algunos aspectos importantes en el último decenio, aunque los progresos no han sido homogéneos, persisten las desigualdades entre mujeres y hombres y sigue habiendo obstáculos importantes, que entrañan graves consecuencias para el bienestar de todos los pueblos,

6. Reconocemos asimismo que esta situación se ha visto agravada por una pobreza cada vez mayor, que afecta a la vida de la mayor parte de la población del mundo y tiene sus orígenes en el ámbito nacional y en el ámbito internacional,

7. Nos comprometemos sin reservas a combatir estas limitaciones y obstáculos y a promover así el adelanto y la potenciación del papel de la mujer en todo el mundo, $y$ convenimos en que esta tarea exige una acción urgente, con espíritu decidido, esperanza, cooperación y solidaridad, ahora y en los albores del nuevo siglo (Organización de las Naciones Unidas, 1995, 2).

Según podemos apreciar, ya desde 1995 la Organización de las Naciones Unidas señalaba los compromisos que debían asumirse para combatir la desigualdad de género y darle a la mujer el lugar que por derecho merece. Estamos hablando de veintidós años en los que si bien se ha avanzado, todavía falta demasiado camino por recorrer para que la igualdad sea un derecho efectivo para las mujeres, más aún cuando estamos viendo en los medios de comunicación cómo la violencia contra las mujeres se ha exacerbado en el último año, mientras que la desigualdad de oportunidades es uno de los principales factores por los cuales es tolerada por parte de las mujeres, al encontrarse en una situación de dependencia económica hacia su agresor. 
En este sentido, en la misma Declaración de Beijing se hizo mención de la necesidad de defender los derechos de las mujeres, así como su dignidad humana, para lo cual se cuenta con diversos instrumentos internacionales, como son la Convención sobre la eliminación de todas las formas de discriminación contra la mujer, la Convención sobre los derechos del niño, la Carta de las Naciones Unidas, la Declaración Universal de los Derechos Humanos, entre otros. En el caso de México, después de la reforma constitucional del 10 de junio de 2011, ya son ley suprema de la nación y deben ser protegidos y respetados por todas las autoridades y proteger de manera efectiva a los principales grupos vulnerables como son las mujeres y las niñas.

Otro punto a resaltar del documento es que "la potenciación del papel de la mujer y la plena participación de la mujer en condiciones de igualdad en todas las esferas de la sociedad, incluidos la participación en los procesos de adopción de decisiones y el acceso al poder, son fundamentales para el logro de la igualdad, el desarrollo y la paz" (Organización de las Naciones Unidas, 1995, 2).

En los últimos años hemos sido testigos de cómo se ha incrementado el número de mujeres presidentas, primeras ministras, Secretarias de Estado o titulares de organismos internacionales. Las mujeres han logrado permear en la política mundial, como sucede con Christine Lagarde, quien fungió como gerente del Fondo Monetario Internacional; Angela Merkel, canciller de Alemania; Theresa May, fue Primera Ministra de Reino Unido; Michelle Bachelet, quien fue dos veces Presidenta de Chile; Nicola Sturgeon, Primera Ministra de Escocia; Ema Solberg, Primera Ministra de Noruega; Beata Szydlo, Primera Ministra de Polonia, Tsai Ing-wen, Presidenta de Taiwan; Sheikh Hasina Wajed, Primera Ministra de Bangladés; Ellen Johnson Sirleaf, Presidenta de Liberia; Marie-Louise Coleiro Preca, Presidenta de Malta; Ameenah Gurib-Fakim, Presidenta de Mauricio; Bidhya Devi Bhandari, Presidenta de Nepal; y Hilda Heine, Presidenta de Islas Marshall, por señalar solamente algunas. 
Lo anterior es un claro reflejo de los avances logrados. Sin embargo, continúan siendo muy pocas las mujeres que llegan a este tipo de cargos. Además, la violencia política que experimentan este tipo de mujeres es muy fuerte y completamente desproporcional a lo que viven los hombres en sus contiendas políticas. A las mujeres se les exige más para que demuestren que son merecedoras de esos cargos.

Otro tema que se abordó en la Declaración de Beijing fue la igualdad de oportunidades y las responsabilidades familiares. Este punto es fundamental para poder consolidar la igualdad, particularmente en el aspecto laboral, ya que la carga del cuidado familiar recae primordialmente en las mujeres, lo que contribuye a que las empresas consideren no contratar a mujeres, o bien darles un trabajo de menor responsabilidad ante las amplias posibilidades de que tengan que ausentarse del trabajo por algún tema familiar, como el cuidado de los hijos. En este sentido, en la Declaración se asentó lo siguiente:

15. La igualdad de derechos, de oportunidades y de acceso a los recursos, la distribución equitativa entre hombres y mujeres de las responsabilidades respecto de la familia y una asociación armoniosa entre ellos son indispensables para su bienestar y el de su familia, así como para la consolidación de la democracia (Organización de las Naciones Unidas, 1995, 2).

Es importante resaltar que la brecha salarial es uno de los principales problemas a los que se enfrentan las mujeres que consiguen acceder al campo laboral. De acuerdo con cifras que se presentaron en el marco de la 61. ${ }^{a}$ sesión de la Comisión de la Condición Jurídica y Social de la Mujer (CSW 61), que se lleva a cabo cada año en la sede de las Naciones Unidas en la ciudad de Nueva York, la brecha salarial entre mujeres y hombres que realizan el mismo trabajo es del 23\% a nivel mundial (cfr. Organización de las Naciones Unidas, 2017):

En promedio, las mujeres ganan 77 centavos por cada dólar que ganan los hombres. En algunos países la brecha es aún 
mayor. Por ejemplo, las mujeres en Suecia y Francia ganan $31 \%$ menos que los hombres. En Alemania esa diferencia es del 49\% y en Turquía del 75\% (Organización de las Naciones Unidas, 2017).

En el año 2014 se lanzó la campaña conocida como He for She, que tuvo como embajadora a la actriz Emma Watson, quien dio un discurso frente a líderes mundiales, pidiendo su ayuda para lograr que los hombres, desde la infancia, se conviertan en defensores del cambio; haciendo énfasis en que la lucha por la igualdad de género ha contribuido a incrementar el odio de los hombres hacia las mujeres. En este sentido, la inclusión de los hombres en este tipo de movimientos sociales es necesaria para erradicar no solo la desigualdad, sino también la violencia de género.

De acuerdo con indicadores con los que cuenta la Organización de las Naciones Unidas a través de la campaña He for She, se sabe que en México, España, Brasil, Perú, Australia, India, Túnez, Rusia, Groenlandia, Italia, Colombia, Ecuador, Chile, Argentina, Paraguay, entre otros, los temas prioritarios en materia de igualdad de género son educación, salud, identidad, trabajo, violencia y política; además, trabajo y violencia son los que mayor preocupación generan (Organización de las Naciones Unidas, 2019). En Estados Unidos, Canadá, China, Japón, Suecia, Finlandia, Reino Unido, entre otros, la principal prioridad es la desigualdad laboral.

Ahora bien, debemos preguntarnos qué podemos esperar de todas estas acciones promovidas por instancias internacionales como la ONU, cuando este organismo ha sido objeto de acusaciones en el sentido de que sus empleadas sufren actos de violencia y que existe impunidad respecto de ellos. En diferentes medios noticiosos se publicó esta situación, exhibiendo a un organismo que tanto ha realizado imputaciones a los Estados para que se promueva la igualdad y se erradique la violencia de género. Se transcriben extractos de una nota periodística que aborda la cuestión: 
Una de las afectadas, que fue violada por un superior, afirmó que pese a presentar evidencias médicas y contar con testigos, una investigación interna concluyó que "no habría pruebas".

La ONU ha permitido la proliferación de casos de acoso y agresión sexual contra sus empleadas en sus oficinas de todo el mundo, en las que se ignora a las víctimas y los agresores operan con "impunidad", según una investigación divulgada por The Guardian.

Este periódico británico entrevistó a docenas de trabajadores y ex empleados de la Organización de Naciones Unidas, quienes describieron una "cultura del silencia" dentro de su estructura y un sistema fallido a la hora de respaldar a las afectadas.

[...]

"Si denuncias, tu carrera prácticamente se acaba, especialmente si eres asesor", indicó de manera anónima una de estas empleadas, que confesó haber sufrido acoso por parte de un supervisor dentro de la organización mientras trabajaba para el Programa Mundial de Alimentos.

Por su parte la ONU ha admitido que considera preocupante el hecho de que incidentes de ese tipo no se lleguen a denunciar, al tiempo que recuerda que el secretario general, António Guterres, "ha dado prioridad a abordar el acoso sexual y a mantener una política de tolerancia cero" contra esas prácticas.

[...]

Una de las afectadas, que denunció que fue violada por un superior del personal de la ONU mientras trabajaba en una localidad remota, afirmó que pese a presentar evidencias médicas y a contar con testimonios de testigos, una investigación interna llevada a cabo por la organización 
concluyó que "no había pruebas suficientes" para respaldar su acusación.

Junto con su empleo, esa mujer perdió además su visado y pasó meses hospitalizada debido al estrés y el trauma de lo ocurrido, según relató al periódico británico.

[...]

The Guardian indica que la ONU ha sido criticada hace tiempo por no investigar de manera adecuada este tipo de hechos y la explotación sexual, perpetrados por sus fuerzas de paz contra aquellos a quienes deben proteger, y grupos de activistas han lamentado la supuesta cultura de impunidad en las oficinas de la organización (EFE, 2018).

Esto evidencia lo que sucede en todo el mundo, en muchas instituciones públicas y privadas; es el vivir a diario de miles de millones de mujeres que deciden o se ven en la necesidad de adentrarse al campo laboral y padecen acoso, hostigamiento o abuso sexual, colocándolas en situación de vulnerabilidad y desventaja para poder acceder a un trabajo digno y bien remunerado, donde puedan vivir en un entorno sin discriminación y sin violencia.

En países como España, donde se han denunciado graves casos de violencia de género, pero también donde podemos observar que hay mujeres dentro del gabinete presidencial ostentando cargos de gran importancia, se han realizado diversas acciones tendentes a combatir la desigualdad y la violencia de género.

Otro país donde se han presentado problemas de igualdad de género es Reino Unido. Aquí las mujeres tienen la obligación de usar tacones para ir a trabajar como parte del código de vestimenta corporativo. Las mujeres que acuden a laborar sin tacones son sancionadas por transgredir ese código. De acuerdo con medios periodísticos "los miembros del Parlamento debatieron una prohibición al uso obligatorio de tacones altos en los centros laborales, en respuesta a una petición iniciada por una recepcionista 
que recibió la orden de regresar a su casa sin derecho a pago por usar zapatos bajos" (Thorp, 2017).

En Estados Unidos de Norteamérica mucho se ha hablado por ejemplo de la desigualdad salarial que padecen las mujeres actrices frente a lo que se les paga a sus compañeros hombres. Sin embargo, actualmente el tema más delicado está en lo referente al acoso sexual que padecen las mujeres; desde los casos denunciados en contra del presidente actual Donald J. Trump, hasta aquellos denunciados mediáticamente contra productores, directores y actores de cine, lo que ha derivado en el movimiento \#MeToo que inició en ese país y que ha sido adoptado internacionalmente.

En Francia, por ejemplo, actualmente se vive un clima de indignación por la postura tomada por personajes del medio artístico como la actriz Catherine Deneuve, quien publicó una carta firmada por otras personalidades en la que criticó fuertemente el movimiento \#MeToo. Las críticas contra la actriz la obligaron a pedir disculpas a las víctimas de la violencia sexual al señalar que "la seducción no es un delito" (Redacción BBC, 2018).

El caso de Catherine Deneuve evidencia cómo las propias mujeres llegan a ser quienes revictimizan a las propias mujeres, y muchas veces se hace desde la ignorancia, lo que impacta en el aspecto de que los actos de molestia se continúen tolerando, así como también la desigualdad de género.

Finalmente, el año 2018 comenzó en cierta forma con buenos avances para el reconocimiento de las mujeres en la economía, como sucedió con la participación de Christine Lagarde en el Foro Económico Mundial. Esta situación representó un gran reconocimiento para la labor que realizan las mujeres en el mundo, ya que en los cuarenta y ocho años de celebrarse este Foro jamás había sido presidido por mujeres. De acuerdo con el diario El País, esta situación acontece derivado de las "fuertes críticas que ha recibido la organización en el pasado ante la escasa presencia de mujeres en la reunión anual, y por otro, a las recetas que la propia 
organización ofrece para reducir la brecha entre hombres y mujeres en el terreno socioeconómico" (González, 2018).

Esta última razón expuesta sobre el ejemplo que debe dar la organización a las demás instancias al ser promotora de "recetas" es de gran importancia ya que, normalmente, las autoridades $u$ organismos que señalan el camino que debe tomarse para generar algún cambio, son quienes no lo implementan al interior de sus estructuras. Todo lo contrario, son señaladas como instancias donde no se respeta lo que se promulga al exterior. En este sentido el Estado, a través de sus distintas instancias administrativas, debe ser el encargado de la observancia del cumplimiento de los tratados internacionales en materia de igualdad de género, siendo el ejemplo hacia el sector privado.

\section{La igualdad de género en México}

El contexto de desigualdad por razones de género que se vive en México, que se ha exacerbado en los últimos años, obliga al Estado a tomar acciones para combatir esta situación que tanto daño le está haciendo a la sociedad; una sociedad que no puede ni debe acostumbrarse o ser inmune a vivir en un entorno donde es normal que a una mujer se le discrimine por el hecho de ser mujer.

Es necesario precisar que la igualdad de género no es una ideología como podrían pensar quienes están en contra de los movimientos sociales que han dirigido esta lucha por la igualdad. Nada más lejos de la realidad. La igualdad de género es un derecho humano que debe respetarse y ser efectivo en todos los ámbitos de la vida pública y privada.

En el caso de México el derecho a la igualdad entre mujeres y hombres lo tenemos reconocido normativamente en el artículo 1, primer y último párrafo, así como artículo 4, primer párrafo, de la Constitución Política de los Estados Unidos Mexicanos, que establecen lo siguiente: 
Artículo $1^{\circ}$. En los Estados Unidos Mexicanos todas las personas gozarán de los derechos humanos reconocidos en esta Constitución y en los tratados internacionales de los que el Estado Mexicano sea parte, así como de las garantías para su protección, cuyo ejercicio no podrá restringirse ni suspenderse, salvo en los casos y bajo las condiciones que esta Constitución establece.

[...]

Queda prohibida toda discriminación motivada por origen étnico o nacional, el género, la condición social, las condiciones de salud, la religión, las opiniones, las preferencias sexuales, el estado civil o cualquier otra que atente contra la dignidad humana y tenga por objeto anularo menoscabar los derechos y libertades de las personas.

Artículo 4. El varón y la mujer son iguales ante la ley. Esta protegerá la organización y el desarrollo de la familia.

Como bien lo demuestra la redacción de los artículos transcritos, la igualdad de género responde a un derecho humano y no a una prerrogativa de los gobiernos, y para hacer eficaz este derecho es necesaria la instrumentación de políticas públicas que promuevan, entre otras cosas, un cambio cultural en hombres y en mujeres para adecuarse a la nueva realidad, que no es más que la posibilidad real y tangible para las mujeres de ocupar espacios que solamente eran accesibles para los hombres.

El mecanismo que tiene el Estado para consolidar la igualdad sustantiva es a través de las políticas públicas. El Plan Nacional de Desarrollo 2013-2018 presentado por el entonces Presidente Enrique Peña Nieto contiene de manera transversal la perspectiva de género en todo el actuar de la Administración Pública Federal; ello significa que en todos los servicios que brinde el Estado a nivel federal deberán considerarse las circunstancias de género para su implementación.

Atendiendo a lo anterior, habría que evaluar si las autoridades están verdaderamente capacitadas para brindar un servicio con 
perspectiva de género, tomando en consideración que un curso de veinte horas no significa que ya se está capacitado en el tema, cuando la costumbre del trabajo de años es mucho más fuerte que una semana al año de sensibilización en el tema. Lo que se quiere resaltar aquí es que es necesario luchar contra la inercia de una cultura machista y patriarcal que se tiene prácticamente desde que nacemos, y que es necesario modificar y conciliar con los nuevos esquemas de igualdad de género.

En los últimos años hemos sido testigos de los altos índices de violencia que experimentan las mujeres en gran parte del mundo, ya sea en países desarrollados como en aquellos en vía de desarrollo como es México y Latinoamérica.

Los medios de comunicación han sido un factor importante para permear en los hogares para ir modificando los anteriores patrones e ir empoderando a las mujeres, haciendo evidente los actos de molestia que experimentan en sus hogares, en el trabajo y en la calle, así como la desigualdad que aún sigue vigente en los distintos ámbitos de la vida y que nos deja ver que la igualdad sustantiva está todavía muy lejos de consolidarse. Los medios de comunicación se han utilizado para fomentar una cultura de igualdad y respeto hacia las mujeres, y han hecho evidente la alta cifra de actos de violencia que ocurren contra las mujeres en México y en distintos lugares del mundo, así como los elevados índices de desigualdad.

En este sentido, el empoderamiento de las mujeres, que se ha llevado a cabo en mayor medida gracias a las acciones que toman muchas organizaciones de la sociedad civil, ha generado lamentablemente un desequilibrio en el sentido de que no se ha trabajado con los hombres para que acepten el nuevo rol femenino, lo que ha derivado en mayores actos de violencia que se ven reflejados en los altos índices de feminicidios o de casos de violencia familiar.

La participación de las organizaciones de la sociedad civil ha sido fundamental para alcanzar los logros que se han obtenido en materia de igualdad de género; pero quien tiene la obligación de incentivar 
estos cambios es el Estado a través de políticas públicas, siendo en el caso de México el Instituto Nacional de las Mujeres la instancia encargada de promover y vigilar el cumplimiento de las políticas de género que deban instrumentar cada uno de los entes del Estado desde el área de su competencia. Esta tarea se lleva a cabo a través de estrategias, líneas de acción y metas establecidas en el Programa Nacional para la Igualdad de Oportunidades y no Discriminación "PROIGUALDAD", estando vigente actualmente el 2013-2018, que obedece a lo contenido en el Plan Nacional de Desarrollo y respecto del cual se cuentan con indicadores de cumplimiento al haberse cumplido la temporalidad de las políticas instrumentadas.

Dentro de las metas nacionales contenidas en el Plan Nacional de Desarrollo se encuentran las de "México en Paz, México Incluyente, México con Educación de Calidad, México Próspero y México con Responsabilidad Global”. Según el Plan, a través de la meta "México en Paz" se buscará la erradicación de la violencia de género; la meta "México Incluyente" hace referencia a disminuir las brechas de desigualdad; la meta "México Próspera" permitirá promover el crecimiento sostenido de la productividad a través de la igualdad de oportunidades (Plan Nacional de Desarrollo, 2013-2018). Si bien no en todas las metas se alude ex profeso a la perspectiva de género, sí se hace referencia a que una de las estrategias transversales es precisamente la perspectiva de género en todas acciones de la administración pública federal (Plan Nacional de Desarrollo, 20132018, 22).

El Plan Nacional de Desarrollo establece en cuanto a la perspectiva de género que

La presente Administración considera fundamental garantizar la igualdad sustantiva de oportunidades entre mujeres y hombres. Es inconcebible aspirar a llevar a México hacia su máximo potencial cuando más de la mitad de su población se enfrenta a brechas de género en todos los ámbitos. Éste es el primer Plan Nacional de Desarrollo que incorpora una perspectiva de género como principio esencial. 
Es decir, que contempla la necesidad de realizar acciones especiales orientadas a garantizar los derechos de las mujeres y evitar que las diferencias de género sean causa de desigualdad, exclusión o discriminación. (Plan Nacional de Desarrollo, 2013-2018, 23).

Si bien, como se afirma en el Plan, este es el primero en incorporar la perspectiva de género, también es cierto que, en la administración de Vicente Fox Quesada (2000-2006), se publicó la Ley General para la Igualdad entre Mujeres y Hombres del 2 de agosto de 2006, lo que significa que llevamos ya once años de su promulgación y los avances aún son mínimos, particularmente en las áreas que son resistentes a entender y aceptar estos cambios.

Ya en el sexenio de Felipe Calderón Hinojosa (2006-2012) la administración pública federal presentó el Programa Nacional para la Igualdad entre Mujeres y Hombres (PROIGUALDAD 2007-2012), a través del cual se requería a las dependencias de la Administración Pública en sus tres órdenes de gobierno, así como a los Poderes de la Unión, que llevaran a cabo acciones que tuvieran como objetivo el desarrollo de las mujeres. Ya desde ese periodo se empezó a contar con presupuestos etiquetados federales dirigidos a la igualdad de género.

El Proigualdad es la política pública de género por excelencia de México. Sus estrategias y líneas de acción son muy ambiciosas; sin embargo, en la práctica puede observarse que queda en simples ambiciones, ya que los mecanismos de control de cumplimiento no cuentan con las herramientas adecuadas para evaluar y sancionar el incumplimiento de las instituciones. El control meramente estriba en exhibir en las reuniones del Sistema Nacional para la Igualdad entre Mujeres y Hombres el grado de cumplimiento de los indicadores, sin evaluar el impacto cualitativo de los mismos. 


\section{Las políticas públicas de igualdad de género en México}

De acuerdo con un estudio publicado por la Comisión Económica para América Latina y el Caribe (CEPAL), realizado en el año 2014,

Las políticas públicas, en la medida en que establecen una obligación de los Estados, son exigibles por parte de la ciudadanía. Hacerlas justas desde un enfoque de igualdad de género las diferencia de otras políticas e impone al Estado nuevos retos en el sentido de considerar la igualdad y la no discriminación en la práctica, reconociendo las necesidades diferenciadas $y$, en consecuencia, las medidas, contenidos y protecciones específicas a través de dichas políticas. Con esta iniciativa se busca, en definitiva, hacer una revisión crítica de las políticas públicas desarrolladas por el Estado y mostrar la medida en que ellas son capaces de transformar situaciones de injusticia de género (Benavente, 2014, 13).

En México, como sucede en muchos países del mundo, los discursos y las normas en materia de igualdad se quedan en anhelos de cambio; no se materializan, y son poco exigidos. Sin embargo, en los últimos años hemos sido testigos de un despertar social que exige por ejemplo justicia para las mujeres víctimas de violencia, pero no así en otros ámbitos como es en la desigualdad salarial, en la vida política, entre otros.

El tema de la violencia se ha exigido, principalmente, a través de manifestaciones, declaraciones en medios de comunicación, entre otros, e incluso ha llegado a instancias internacionales como la Corte Interamericana de Derechos Humanos con el caso del Campo Algodonero (caso González y otras v. México, sentencia dictada el 16 de noviembre de 2009), cuya resolución obligó al Estado Mexicano a tipificar el homicidio de mujeres por razones de género con un tipo penal muy específico, el del femicidio. Este es un claro ejemplo de la 
exigencia del cumplimiento de los derechos de las mujeres, pero son pocos los casos que han visibilizado un verdadero despertar de las instituciones y de la sociedad en su conjunto.

Mundialmente nos estamos enfrentando a un proceso de emancipación de las mujeres, particularmente las provenientes de las nuevas generaciones; generaciones que cuentan con mayor nivel de estudios, un panorama más preciso de lo que buscan en la vida y exigencias sociales que harán al Estado para que este haga su trabajo de garantizar su desarrollo de manera igualitaria.

Según Anne Goetz, la justicia de género "puede definirse entonces como el logro de la igualdad entre mujeres y hombres en conjunto con las medidas para reparar las desventajas que llevan a la subordinación de las mujeres y para permitirles acceder y controlar los recursos en combinación con la agencia humana" (Goetz, 2014, 16).

En otros ámbitos, como por ejemplo en el laboral, donde gran número de mujeres sufren discriminación, así como acoso $\mathrm{y}$ hostigamiento laboral y sexual, no se han visibilizado en gran magnitud cambios cualitativos por la consumación de políticas públicas.

Como bien apuntan María Cristina Benavente y Alejandra Valdés, "la búsqueda de la justicia de género debe entonces apuntar en sus fundamentos a defender una política social de la igualdad y una política cultural de la diferencia, en el entendido de que en la mayoría de las injusticias se combinan injusticias de redistribución y de reconocimiento" (Benavente, 2014, 17).

Ahora bien, tenemos políticas públicas, instituciones, presupuestos y normatividad, pero ¿qué está faltando?, ¿en que se está equivocando el Estado?, ¿qué es lo que el Estado no alcanza a ver?, ¿por qué no hay evidencias de los resultados que sean tangibles y no solamente cifras frías presentadas en los informes de las autoridades? 
En este sentido, tenemos que analizar la "capacidad" que tiene el Estado para hacer frente a estas condiciones de desigualdad, que han llevado incluso a incrementar los índices de violencia que experimentan las mujeres en su hogar, en la vía pública, en el trabajo y en los servicios que presta el Estado.

Esta capacidad a la que hacemos referencia ha sido objeto de análisis en el mundo académico, que la ha considerado como un instrumento modernizador.

Hacia fines de siglo $X X$, voces académicas, gubernamentales y de organizaciones de consultoría manifestaron la necesidad de una segunda generación de reformas al Estado, apuntando más a la "modernización" del Estado que a su desmantelamiento (Gacía Delgado, 2001; Rabotnikiv, 1999; Bresser Pereira, 1998). A partir de entonces tomó impulso el concepto "capacidades institucionales del Estado", siendo postulado como variable relevante para la calidad de las políticas públicas (Rey, 2014, 118).

Esta falta de modernización y continuo desmantelamiento del Estado es lo que principalmente lleva al fracaso de las políticas públicas. Se da, en particular, por los cambios de gobierno, por el sello que cada líder de una Nación quiere dejar; esto lleva a que intente borrar los aciertos de un líder anterior que pudieran arrojar sombra a lo que pretenda alcanzar con políticas públicas mal diseñadas y un Estado desmantelado e inútil institucionalmente, como está sucediendo en el caso de México con la eliminación de programas sociales que apoyaban a mujeres en situación de vulnerabilidad.

En este contexto, con respecto al primer interrogante planteado más arriba, podríamos decir que ha faltado una labor debidamente coordinada entre Estado y sociedad. El Estado no puede asumir el control total que implica un cambio cultural y social, necesita forzosamente de la participación de la sociedad. México ha vivido a lo largo de su historia un sistema eminentemente patriarcal, donde la participación de la mujer se enfocaba al cuidado del hogar y no a 
actividades económicas. En este sentido, es necesario que el Estado promueva campañas de sensibilización para hombres y mujeres entendiendo los nuevos roles. Como bien lo señala la campaña HeforShe, se necesita que los hombres se involucren, que sean aliados del cambio.

En relación con las fallas del Estado, principalmente se deben a la falta de continuidad en algunas políticas públicas, de conocimiento por parte de las autoridades de lo que significa la perspectiva de género y lo que implica su instrumentación, y de controles eficaces de seguimiento de las políticas y del ejercicio presupuestario. Debemos señalar que el Estado debe ser ejemplo de la sociedad para generar este cambio, no actuar meramente como quien proporciona las ideas o publica las leyes, sino como quien tiene la obligación de implementarlas realmente en su actuar y no solamente de manera discursiva.

Respecto al interrogante sobre aquello que el Estado no alcanza a ver, podríamos decir que el Estado no se ha involucrado verdaderamente en generar ese cambio. Pareciera que solamente reacciona cuando se presenta alguna advertencia internacional; cuando ya existen movimientos sociales ante el hartazgo social por la negligencia, insensibilidad, intransigencia e ignorancia de muchos servidores públicos; o bien, cuando los servidores públicos omiten cumplir sus funciones, situación que afecta la vida pública y privada de las mujeres.

Finalmente, sobre la pregunta acerca de la falta de evidencia tangible de los resultados, las instituciones que tienen por mandato realizar acciones en materia de igualdad de género — de acuerdo con las líneas de acción previstas en el Proigualdad, y además de contar con presupuesto etiquetado en el Presupuesto de Egresos de la Federación - no cuentan con mecanismos para medir el impacto de sus acciones. En muchos de los casos, no hay un seguimiento real de los cambios que se generan, lo cual puede deberse en parte a que no se cuenta con una planificación adecuada. Debe trabajare en primera 
instancia con grupos focales e ir incrementando paulatinamente la población meta, y ya con resultados tangibles.

El Instituto Nacional de las Mujeres, instancia encargada de impulsar el Proigualdad y de coordinar las acciones entre las dependencias, cuenta con un mecanismo de seguimiento que presenta los avances en el cumplimiento de los indicadores por objetivo. Los que se exponen a continuación son los resultados más actuales que presenta INMUJERES en su página web. Esta información siempre debe estar al alcance de la sociedad para que esté informada y le pueda exigir cuentas a las autoridades, así como también para que se vayan sensibilizando, aun cuando solamente vemos cifras frías y no nos enteramos realmente de lo que padece una mujer que ha vivido discriminación y violencia por el simple hecho de ser mujer.

A continuación, se presentan unas gráficas de cumplimiento de indicadores por objetivo (PROIGUALDAD). ${ }^{2}$

Objetivo Transversal 1: Alcanzar la igualdad sustantiva entre mujeres y hombres, y propiciar un cambio cultural respetuoso de los derechos de las mujeres.

Índice de avance en la armonización legislativa por entidad federativa, Nacional (2010-2018):

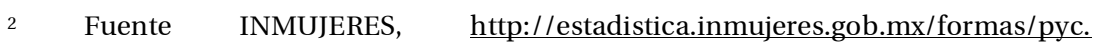
php?IDPrograma=1, consultado el 8 de enero de 2020. 


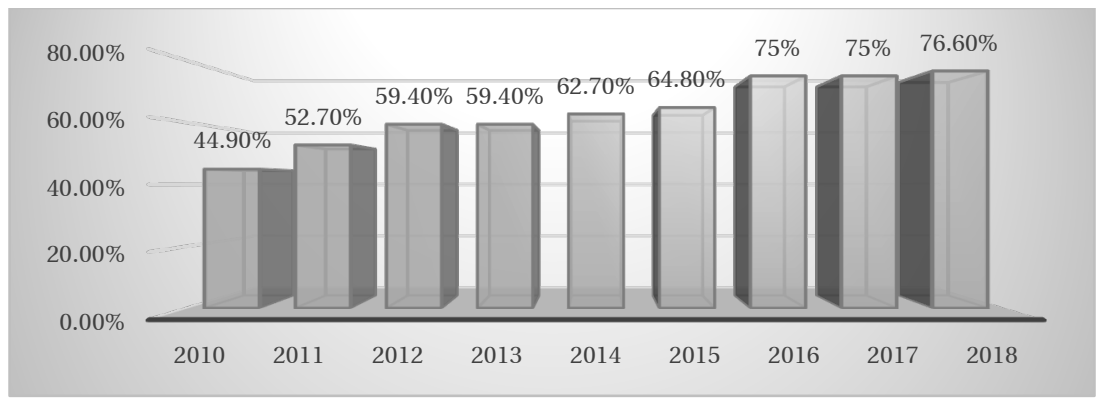

Porcentaje de mujeres en puestos directivos de la administración pública (2010-2019):

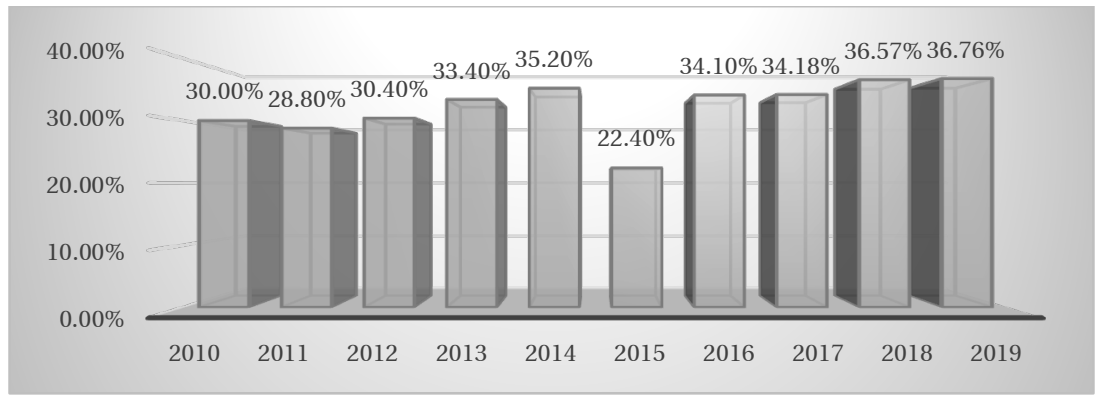

Porcentaje de mujeres en cargos de elección popular en municipios, Participación política (2010-2019):

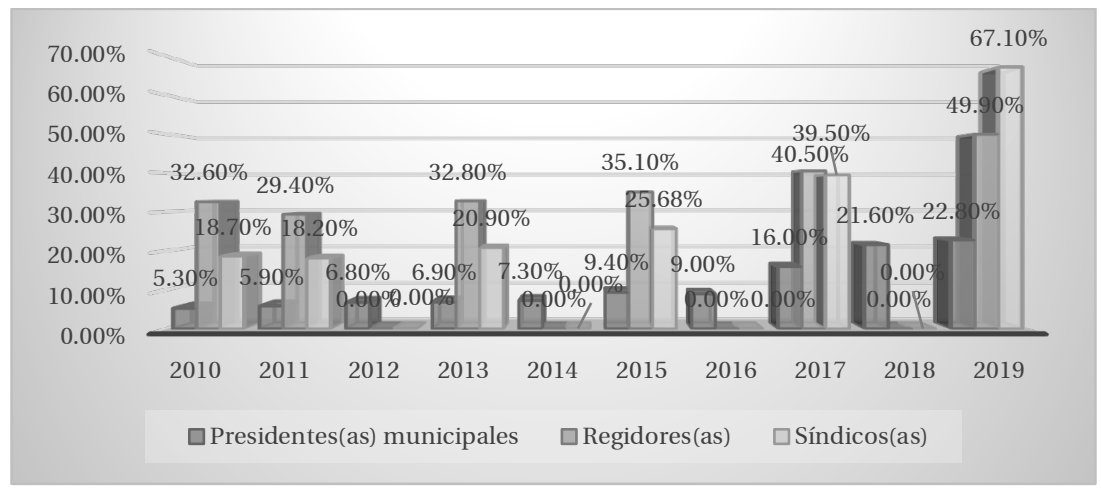


Revista Internacional de Derechos Humanos / ISSN 2250-5210 / 2020 Vol. 10, No. 1 revistaidh.org

Objetivo transversal 2: Prevenir, atender, sancionar y erradicar la violencia contra mujeres y niñas, y garantizarles acceso a una justicia efectiva.

Mujeres víctimas de violencia atendidas en refugios y centros de justicia (2011-2018):

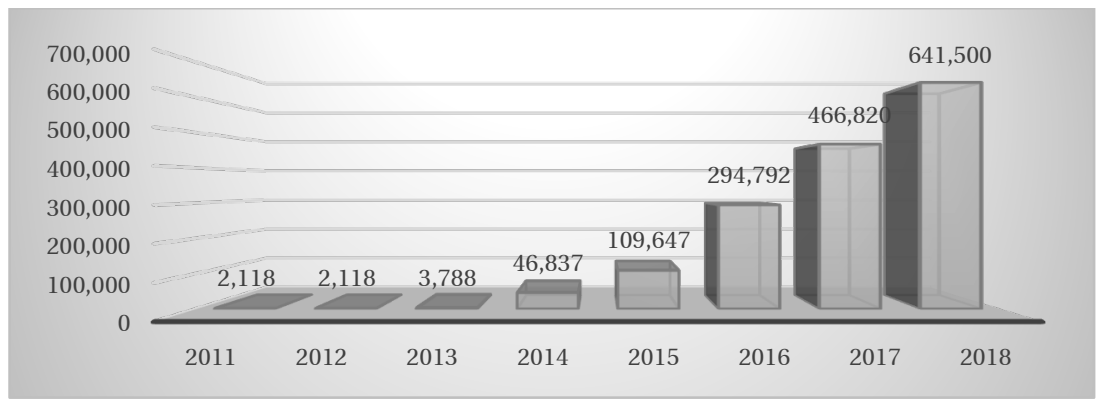

Delitos contra mujeres ingresados en los Tribunales Superiores de Justicia Estatales, Nacional (2012-2018):

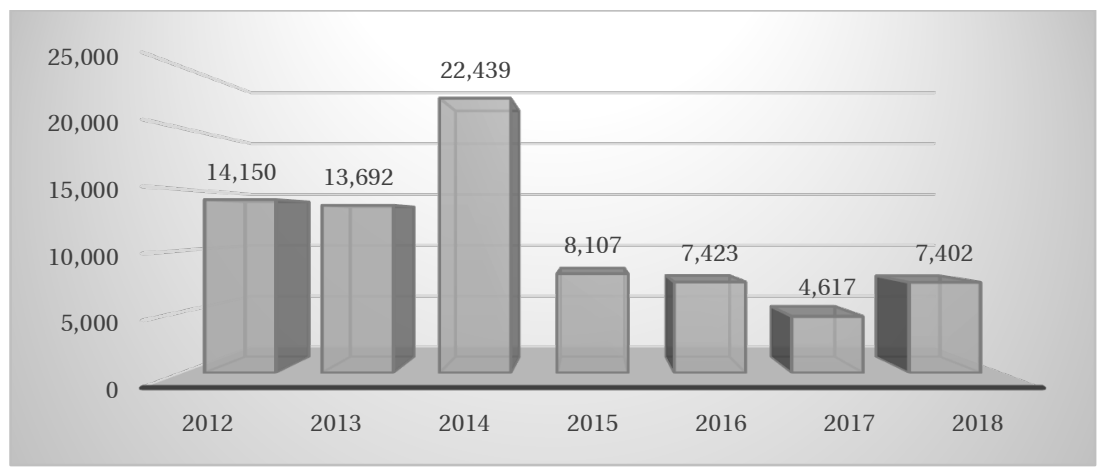

Denuncias presentadas ante agencias del Ministerio Público por delitos de género (2010-2018): 


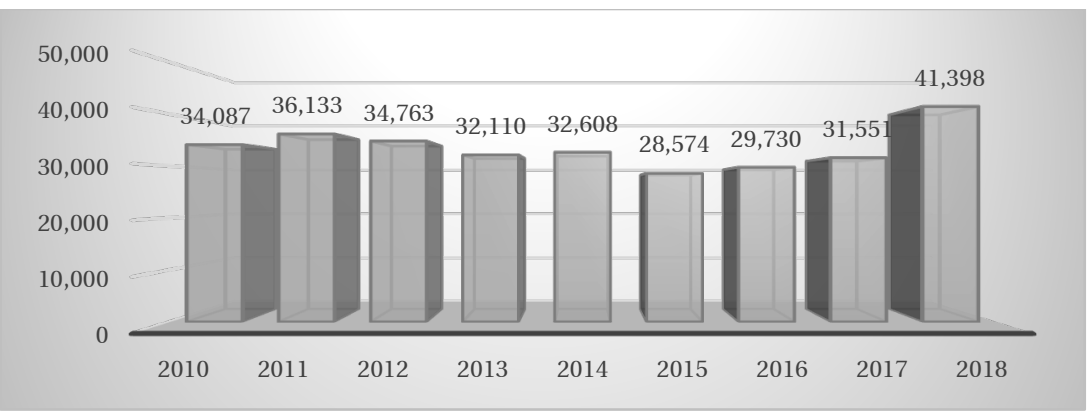

Objetivo transversal 3: Promover el acceso de las mujeres al trabajo remunerado, empleo decente y recursos productivos, en un marco de igualdad.

Tasa de participación femenina de 14 años y más en el mercado de trabajo (2010-2019):

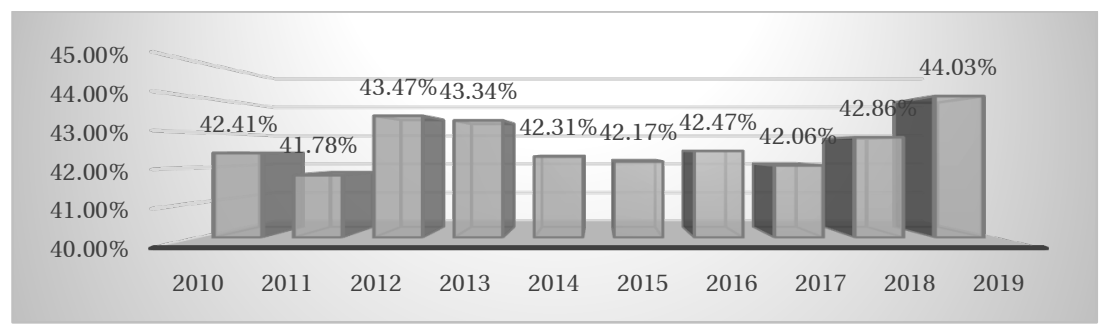

Índice de discriminación salarial por sector de actividad (2010-2019):

\begin{tabular}{|l|l|l|l|l|l|l|l|l|l|l|}
\cline { 2 - 10 } \multicolumn{1}{c|}{} & $\mathbf{2 0 1 0}$ & $\mathbf{2 0 1 1}$ & $\mathbf{2 0 1 2}$ & $\mathbf{2 0 1 3}$ & $\mathbf{2 0 1 4}$ & $\mathbf{2 0 1 5}$ & $\mathbf{2 0 1 6}$ & $\mathbf{2 0 1 7}$ & $\mathbf{2 0 1 8}$ & $\mathbf{2 0 1 9}$ \\
\hline Total & $\mathbf{- 8 . 3 3}$ & $-\mathbf{5 . 2 9}$ & $\mathbf{- 4 . 6 5}$ & $\mathbf{- 3 . 1 0}$ & -6.38 & -6.19 & -5.17 & -5.90 & $\mathbf{- 5 . 3 0}$ & $-\mathbf{6 . 6 2}$ \\
\hline $\begin{array}{l}\text { Actividades } \\
\text { agropecuarias }\end{array}$ & -0.20 & -3.79 & -0.96 & 2.96 & 1.16 & -1.48 & -3.16 & 14.60 & 2.80 & 1.21 \\
\hline Comercio & -25.40 & -21.33 & -19.27 & -19.21 & -17.44 & -23.80 & -24.29 & -16.90 & -18.40 & -22.83 \\
\hline $\begin{array}{l}\text { Restaurantes y } \\
\text { servicios de } \\
\text { alojamiento }\end{array}$ & -13.20 & -3.64 & -7.64 & -2.06 & -18.21 & -3.86 & -4.62 & -10.40 & -5.70 & -1.04 \\
\hline $\begin{array}{l}\text { Industria } \\
\text { manufacturera }\end{array}$ & -22.10 & -15.85 & -17.60 & -18.09 & -18.25 & -17.77 & -22.30 & -17.80 & -15.20 & -18.21 \\
\hline $\begin{array}{l}\text { Servicios } \\
\text { profesionales, }\end{array}$ & -37.90 & -10.89 & -6.03 & -7.89 & -6.82 & -14.11 & -5.97 & -3.50 & -6.70 & -8.71 \\
\hline
\end{tabular}


Revista Internacional de Derechos Humanos / ISSN 2250-5210 / 2020 Vol. 10, No. 1 revistaidh.org

\begin{tabular}{|c|c|c|c|c|c|c|c|c|c|c|}
\hline $\begin{array}{l}\text { financieros y } \\
\text { corporativos }\end{array}$ & & & & & & & & & & \\
\hline $\begin{array}{l}\text { Servicios } \\
\text { sociales }\end{array}$ & -13.90 & -9.50 & -11.10 & -9.56 & -14.76 & -15.23 & -6.94 & -13.30 & -11.70 & -11.71 \\
\hline $\begin{array}{l}\text { Servicios } \\
\text { diversos }\end{array}$ & 3.10 & 8.73 & -0.97 & 3.72 & -2.20 & 0.16 & 0.16 & 1.20 & 3.80 & 6.56 \\
\hline Construcción & -12.30 & -31.72 & -15.19 & 6.20 & -19.59 & -17.07 & -13.04 & -10.50 & -11.10 & -6.44 \\
\hline $\begin{array}{l}\text { Industria } \\
\text { extractiva y de la } \\
\text { electricidad }\end{array}$ & 20.74 & -8.47 & 8.53 & -1.32 & -1.80 & -7.08 & 2.72 & 1.70 & 8.90 & -13.40 \\
\hline $\begin{array}{l}\text { Transportes, } \\
\text { comunicaciones, } \\
\text { correo y } \\
\text { almacenamiento }\end{array}$ & 2.80 & -0.32 & -3.41 & -9.16 & 4.08 & -3.63 & 1.86 & 0.90 & 3.60 & -3.63 \\
\hline $\begin{array}{l}\text { Actividades del } \\
\text { gobierno y de } \\
\text { organismos } \\
\text { internacionales } \\
\text { y extraterrito- } \\
\text { riales }\end{array}$ & -2.70 & -1.51 & 2.24 & 1.57 & -0.60 & 2.58 & 3.89 & 2.10 & -5.90 & -0.89 \\
\hline
\end{tabular}

Objetivo transversal 6: Incorporar las políticas de igualdad de género en los tres órdenes de gobierno y fortalecer su institucionalización en la cultura organizacional.

Número de funcionarias y funcionarios capacitados y certificados en igualdad de género (2012-2018):

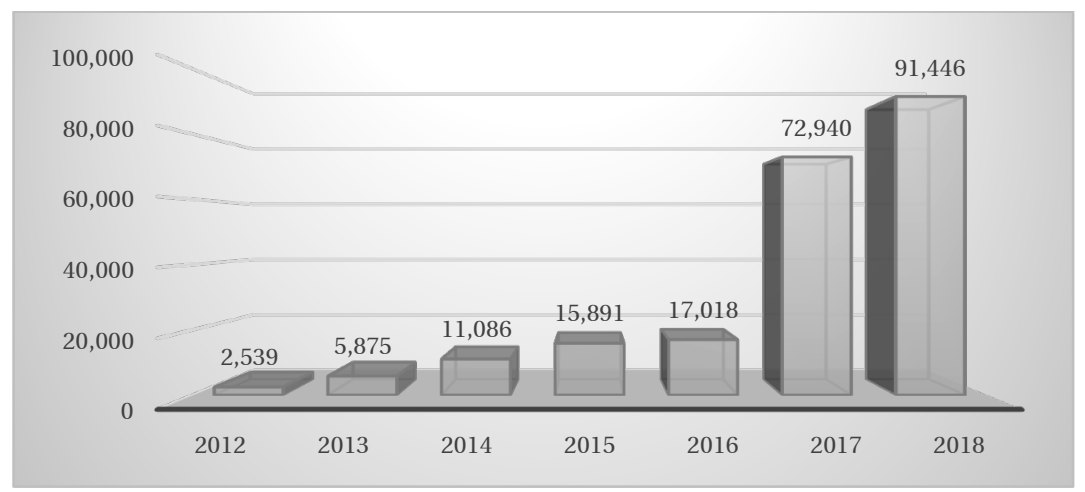


En estos datos, puede verse de manera preocupante el número de mujeres que han tenido que acudir a refugios y centros de justicia para mujeres. El incremento de 2011 y 2012 frente a 2015, y aún más en los tres años subsecuentes 2018, es alarmante y el Estado está en la obligación de actuar para disminuir y erradicar la violencia de género. Las políticas están fallando en este sentido y las cifras lo demuestran.

Sobre estas cifras, sería importante conocer si los elevados números se deben a que las mujeres se han empoderado y están más informadas sobre este tipo de servicios, o se deben a un incremento en la violencia que sufren. La cifra de mujeres que acuden en busca de estos servicios contrasta con el número de denuncias presentadas ante agentes del Ministerio Público, lo que evidencia la falta de confianza de las mujeres para acudir con estos servidores públicos, ante la revictimización de la que son objeto generalmente por la discriminación de género.

De igual forma, se puede observar que la desigualdad salarial continúa siendo una constante en la vida laboral de las mujeres, por lo que es necesario realizar cambios en las políticas públicas en esta materia, ya que como se refleja en las gráficas, casi el $50 \%$ de la población femenina se encuentra activa en el mercado laboral.

\section{Conclusiones}

De todo lo expuesto podemos advertir que las políticas públicas que ha debido instrumentar el Estado - prácticamente de manera obligatoria ante la continua y creciente desigualdad que existe entre mujeres y hombres en distintos ámbitos-, si bien han obtenido algunos avances en pro de la igualdad sustantiva, requieren aún demasiado por hacer.

El Estado continúa en deuda con las mujeres, quienes se ven obligadas en ciertas ocasiones a tomar acciones personales para salvaguardar su integridad, o bien a relegarse aún más por temor a las consecuencias privadas y sociales, como sería el abandono de su 
cónyuge ante el reclamo de haber preferido tener un futuro profesional en lugar de atender a su familia, sumado a una recriminación social por supuestamente querer involucrarse en el ámbito masculino del trabajo.

Los altos índices de violencia son en gran parte consecuencia de la desigualdad de género, derivada de la ignorancia, tanto de hombres como de mujeres, sobre lo que implica la igualdad de género y lo importante, justo y necesario que es que las mujeres puedan desarrollarse en todos los ámbitos sin tener que padecer críticas, abandono familiar o incluso la muerte.

En este sentido, la evaluación de las políticas públicas, aunque de cierta forma deficiente - al solo presentar cifras que no reflejan un cambio real de conciencia en la sociedad- requiere de una nueva dirección. También es urgente una modificación en las políticas públicas de igualdad de género, así como de prevención y de erradicación de la violencia contra las mujeres. Todos los días, en muchos lugares del mundo escuchamos y vemos una ola creciente de denuncias sociales, que exigen un cambio a sus autoridades y al resto de la sociedad, para que se involucre en un problema que es de todas y todos.

Como sociedad no podemos ser apáticos, y mucho menos acostumbrarnos a este tipo de problemáticas. Si no se atiende de manera efectiva, costará muchas generaciones alcanzar un cambio mínimo. Los derechos de las mujeres no son un capricho; su respeto y eficacia es una obligación para el Estado.

En este sentido, para avanzar en la igualdad sustantiva es necesario un cambio cultural, que debe ir instrumentado desde la educación básica hasta la superior. Esto quiere decir que es necesario transversalizar la perspectiva de género en todos los planes de estudio, así como en el sector laboral. Sin embargo, es el Estado, a través de sus instituciones, quien debe dar el ejemplo al sector privado, y promover estímulos fiscales a las empresas que estén generando un cambio cultural en el ámbito laboral a favor de la 
igualdad. Finalmente, se debe realizar una revisión y valoración plena de los operadores de justicia, quienes carecen de formación para juzgar con perspectiva de género, dejando desamparadas a muchas mujeres.

\section{Referencias bibliográficas}

Benavente R, María Cristina y Valdés B., Alejandra, Políticas públicas para la igualdad de género. Un aporte a la autonomía de las mujeres, Santiago de Chile, Comisión Económica para América Latina y el Caribe (CEPAL), octubre de 2014.

Benavente R, María Cristina y Valdés B., Alejandra, Políticas públicas para la igualdad de género. Un aporte a la autonomía de las mujeres, Santiago de Chile, Comisión Económica para América Latina y el Caribe (CEPAL), octubre de 2014.

Burgoa Toledo, Carlos Alberto, Finanzas públicas, en Derecho Administrativo, México, Editorial Porrúa, 2014.

Centro de noticias ONU, ONUMujeres afirma que la brecha salarial del 23\% entre mujeres y hombres es un robo, Organización de las Naciones Unidas, 14 de marzo de 2017, http://www.un.org/sustainabledevelopment/es/2017/03/onumujeres-afirma-que-la-brecha-salarial-del-23-entre-mujeres-yhombres-es-un-robo/

Constitución Política de los Estados Unidos Mexicanos.

EFE, Acusan a la ONU de ocultar los abusos sexuales contra sus empleadas, Periódico El Mundo, España, 19 de enero de 2018, http://www.elmundo.es/internacional/2018/01/19/5a61da9026 8e3eb4388b4754.html

Fraser, Nancy, Multiculturalidad y equidad entre los género: un nuevo examen de los debates en torno a la $<<$ diferencia $>>$ en EE.UU., Revista de Occidente, España, 1995, http://www.mujeresenred.net/IMG/pdf/Multiculturalidad_y_e quidad_entre_los_generos.pdf

Goetz, Anne en Benavente R, María Cristina y Valdés B., Alejandra, Políticas públicas para la igualdad de género. Un aporte a la autonomía de las mujeres, Santiago de Chile, Comisión Económica para América Latina y el Caribe (CEPAL), octubre de 2014. 
González, Alicia, Foro Económico Mundial de 2018: El momento de la "mujer de Davos", España, Periódico El País, 22 de enero de 2018, https://elpais.com/economia/2018/01/21/actualidad/15165458 46_483897.html.

Ley del Instituto Nacional de las Mujeres, México, 2015.

Ley General para la Igualdad entre Mujeres y Hombres, México, 2016.

Meny, Yves y Thoenig, Jean-Claude, Las políticas públicas, Barcelona, Editorial Ariel, S.A., 1992.

Millet, Kate, Política sexual, Ediciones Cátedra, Universitat de Valencia, Instituto de la Mujer, España, 1970, https://revistaemancipa.org/wpcontent/uploads/2017/09/Kate-Millett-Politica-sexual.pdf

Organización de las Naciones Unidas, Declaración y Plataforma de Acción de Beijing, Cuarta Conferencia Mundial sobre la Mujer, Beijing, 1995.

Organización de las Naciones Unidas, He for She, Track Your Impact, 2019, http:/ / www.heforshe.org/en

Oszlak, Oscar y O’Donnell Guillermo, Estado y políticas estatales en América Latina: hacia una estrategia de investigación, Redes, Vol. 2, núm. 4, Buenos Aires, Argentina, Universidad Nacional de Quilmes, 1995, pp. 99-128.

Pateman, Carole, El contrato sexual, México, Editorial UAM y ANTROPOS Editorial del Hombre, 1995, https://jcguanche.files.wordpress.com/2014/01/131498859carole-pateman-el-contrato-sexual-1995.pdf

Plan Nacional de Desarrollo 2013-2018, México, http://itcampeche.edu.mx/wp-content/uploads/2016/06/PlanNacional-de-Desarrollo-PND-2013-2018-PDF.pdf.

Redacción, La actriz francesa Catherine Deneuve pide perdón a las víctimas de acoso tras la polémica levantada por sus críticas a \#MeToo, BBC Mundo, 15 de enero de 2018, http://www.bbc.com/mundo/noticias-42688199.

Rey, Maximiliano, Capacidad estatal y poder del Estado en Latinoamérica del siglo XXI: Una perspectiva política para el análisis de las políticas públicas y la estatalidad, Revista Estado y Políticas Públicas, No. 2, Argentina, 2014, 
https://revistaeypp.flacso.org.ar/files/revistas/1401638855_arti culo-1.pdf

Ridgeway, C.L y Correll, S.J., Unpacking the Gender System. A Theoretical Perspective on Gender Beliefs and Social Relations, Gender and Society, Vol. 18, 2004.

Rodríguez Gustá, Ana Laura, Las políticas sensibles al género: variedades conceptuales y desafios de intervención, Argentina, Universidad Nacional de San Martín, 2008.

Stein, Ernesto y Tommasi, Mariano, La política de las políticas públicas, en Política y gobierno, Vol. XIII, Núm. 2, II Semestre de 2006, p. 395, http://repositoriodigital.cide.edu/bitstream/handle/11651/1876/286-524-1PB.pdf?sequence $=1$

Thorp, Nicola, Tacones, el debate laboral en Reino Unido, El Financiero, México, sección Mundo, 6 de marzo de 2017, http:/ / www.elfinanciero.com.mx/mundo/tacones-el-debatelaboral-en-reino-unido.html. 